\title{
Ulceration of the small intestine in children with coeliac disease
}

\author{
M Eltumi, $M$ J Brueton, N Francis
}

\begin{abstract}
Background-Ulceration of the small intestine in children has not been previously described.

Patients-Two children, aged 12 and 18 months, presented with a history of failure to thrive and intractable diarrhoea.

Results and Conclusions-Upper gastrointestinal endoscopy showed multiple ulcers involving the first and second parts of the duodenum. Histology of biopsy specimens taken from these sites confirmed ulceration and showed other features consistent with a diagnosis of coeliac disease. They both showed pronounced clinical improvement and satisfactory linear growth on a gluten free diet. A year later the diagnosis of coeliac disease was confirmed on a biopsy controlled gluten challenge, and repeat endoscopy showed complete resolution of the intestinal ulceration.

(Gut 1996; 39: 613-614)
\end{abstract}

Keywords: coealiac disease, intestinal ulceration, endoscopy.

An association between ulceration of the small intestine and coeliac disease was first reported in 1967 by Bayless et al. ${ }^{1}$ The authors described an adult patient who presented with an acute abdomen and on laparotomy was found to have ulceration in the duodenum, jejunum, and ileum. Further adult patients were described ${ }^{2-4}$ in whom ulceration was associated with other disorders of the small bowel such as unclassified sprue, lymphomas, and histiocytosis leading to malabsorption. ${ }^{5-8}$ Other features of the patients' presentation included an acute abdomen, perforation, haemorrhage, or stricture formation and obstruction; there was a mortality of over $70 \%$. The diagnosis of ulceration of the small intestine was usually established at laparotomy, and in some cases by contrast radiography. Multiple ulcers were seen, predominantly involving the jejunum and ileum. In patients with coeliac disease the ulcers did not improve on a gluten free diet or corticosteroid treatment. ${ }^{78}$ Increasingly ulceration and stricture formation in coeliac disease have been associated with enteropathy associated lymphoma ${ }^{5}$ to the extent that a picture of adult coeliac disease with ulceration should stimulate a thorough search for underlying lymphoma. In children coeliac disease is a common cause of malabsorption, but ulceration of the small intestine has not been described. We report two children with this association who did not have any underlying disorder.

\section{Case histories}

Case I was a white female child who presented at 18 months of age with a three week history of vomiting, diarrhoea, weight loss, and abdominal distension. She had no previous history of illness. The neonatal period was normal. She had been breast fed until nine months, when solid foods were first introduced. She was the only child of healthy unrelated parents. Her uncle had coeliac disease and her aunt had died of cystic fibrosis. At presentation, she was unwell with pronounced abdominal distension and buttock wasting.

Case II was a one year old male Asian child who presented with a four month history of diarrhoea, recurrent vomiting, and weight loss. He had had no relevant illnesses in the past. The neonatal period was normal. He had been breast fed for the first two weeks of life, and then started on a cows' milk formula. Commercially available baby foods were introduced at the age of three months. There were no relevant family illnesses. At presentation he was thin and miserable, with abdominal distension and buttock wasting.

\section{Investigation and follow up}

The antigliadin, antireticulin, and antiendomesium antibodies were positive in both patients. The serum albumin concentrations were 28 and $37 \mathrm{~g} / \mathrm{l}, \mathrm{IgA}$ concentrations 1.2 and $1.0 \mathrm{~g} / \mathrm{l}$, and haemoglobin concentrations 13 and $12 \mathrm{~g} / \mathrm{dl}$ in cases I and II respectively. Ferritin, red cell folate, clinical chemistry, and IgG and IgM concentrations were all normal and stool microbiology showed no pathogens. Small bowel biopsy specimens were obtained endoscopically, macroscopically there was inflammation of the entire duodenal mucosa with multiple ulcers, mainly involving the first and second parts of the duodenum. Multiple biopsies from the distal part of the duodenum showed mucosal erosions, villous atrophy, crypt hyperplasia, and an increase in the number of intraepithelial lymphocytes; the histological features were suggestive of coeliac disease (Figure).

Both patients were started on a gluten free diet. Their symptoms resolved and they showed significant catch up growth in weight and length. A year later, while on a gluten free diet, upper gastrointestinal endoscopy showed normal duodenal mucosa and biopsy specimens from the distal part of the duodenum were normal in both patients. On gluten challenge, case I developed diarrhoea, anorexia, and weight loss, and the gluten containing diet was discontinued after four weeks when further biopsy specimens were taken; they 


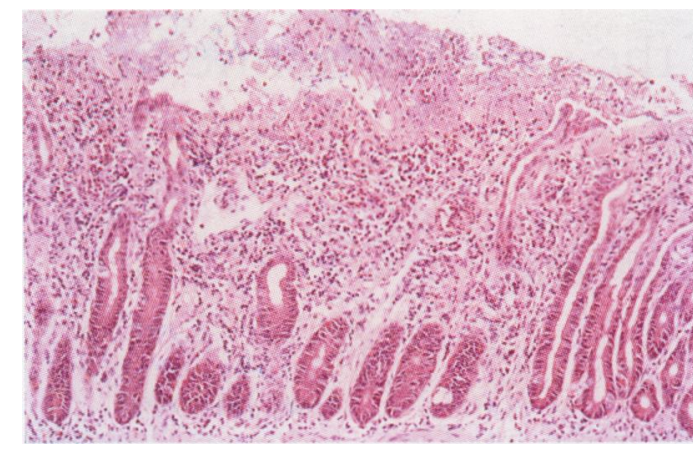

Endoscopic small bowel biopsy specimen showing superficial erosion and loss of villus architecture and accompanying crypt hyperplasia.

showed villous atrophy. Case II was able to tolerate a gluten containing diet and after three months the postchallenge biopsy specimens from the distal part of the duodenum confirmed the diagnosis of coeliac disease. No intestinal ulceration was seen in either patient. Both have remained well with a normal growth rate over a three year follow up period on a gluten free diet.

\section{Discussion}

The relation of ulcerative jejunoileitis to enteropathy associated $T$ cell lymphoma in adult patients is well established. ${ }^{569}$ This association is so strong that it has been suggested that 'benign' ulcerative jejunoileitis usually represents a manifestation of lymphoma with secondary inflammation obscuring the nature of the malignancy. In our patients, ulceration of intestinal flat mucosa occurred in the absence of lymphoma. Ulceration of the small intestine in children with coeliac disease has not been previously reported and this manifes- tation may now be considered as one of the complications or variants of the presentation of coeliac disease. The relevance of the features seen in these cases is difficult to establish as this is primarily a macroscopic appearance which would not have been identified so readily in the past using the technique of peroral capsule biopsy.

The clinical course of these coeliac children with ulceration of the distal duodenum was different from that described in adult patients. In the adults there is some heterogeneity, but there was a notably poor response to gluten exclusion. ${ }^{78}$ It has been suggested that a prolonged alteration in enterocyte turnover could result in local areas of regenerative failure ${ }^{1}$; however, in some adults the ulcers persisted despite the recovery of normal villous architecture. The aetiology of ulceration in the small bowel in coeliac disease remains uncertain, and its importance in relation to other aspects of this disorder remains to be established.

1 Bayless TM, Kepeowitz RF, Shelley WM, Ballinger WF, Hendrix TR. Intestinal ulceration - A complication of coeliac disease. N Engl f Med 1967; 276: 996-1002.

2 Jones PE, Gleeson MH. Mucosal ulceration and mesenteric lymphadenopathy in coeliac disease. BMF 1973; 3: 212-3.

3 Venturatos SG, Hines CJR, Blalock JB. Ulceration of the small intestine in a patient with coeliac disease. South Med f 1984; 77: 520-2.

4 Kiely PB, O'Donovan PJ, O'Donovan PA. Benign ulceration, perforation and intussusception in coeliac disease. $\mathfrak{f}$ Coll Surg Edinb 1985; 30: 272-3.

5 Isaacson P, Wright DH. Malignant histocytosis. Its relationship to malabsorption and ulcerative jejunitis. Hum Pathol ship to malabsorp

6 Isaacson $\mathrm{P}$, Wright $\mathrm{DH}$. Intestinal lymphoma associated with malabsorption. Lancet 1978; i: 67-70.

7 Baer AN, Bayless TM, Yardley JH. Intestinal ulceration and malabsorption syndromes. Gastroenterology 1980; 79: 754-65.

8 Robertson DAF, Dixon MF, Scott BB, Simpson FG, Losowsky MS. Small intestinal ulceration: diagnostic

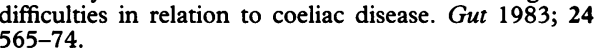

9 Mills PR, Brown IL, Watkinson G. Idiopathic ulcerative enteritis. Report of five cases and review of literature. QfM 1980; 49: 133-49. 